\title{
XMM detection of the supernova remnant G337.2+0.1
}

\author{
Jorge A. Combi ${ }^{1}$ \\ Departamento de Física (EPS), Universidad de Jaén, Campus Las Lagunillas s/n, Ed-A3, \\ 23071 Jaén, Spain \\ jcombi@ujaen.es \\ Juan F. Albacete Colombo ${ }^{2}$ \\ Osservatorio Astronomico di Palermo, Piazza del Parlamento 1, Palermo (90141), Italy \\ Gustavo E. Romero ${ }^{2,4}$ and Paula Benaglia ${ }^{3,4}$ \\ Facultad de Ciencias Astronómicas y Geofísicas UNLP, Paseo del Bosque, B1900FWA La \\ Plata, Argentina \\ Instituto Argentino de Radioastronomía, C.C.5, (1894) Villa Elisa, Buenos Aires, \\ Argentina
}

\begin{abstract}
We report the first XMM detection of the SNR candidate G337.2+0.1 (=AX J1635.9-4719). The object shows centrally filled and diffuse X-ray emission. The emission peaks in the hard 3.0-10.0 keV band. A spatially resolved spectral study confirms that the column density of the central part of the SNR is about $\mathrm{N}_{H} \sim 5.9( \pm 1.5) \times 10^{22} \mathrm{~cm}^{-2}$ and its X-ray spectrum is well represented by a single power-law with a photon index $\Gamma=0.96 \pm 0.56$. The non-detection of line emission in the central spectrum is consistent with synchrotron radiation from a population of relativistic electrons. Detailed spectral analysis indicates that the outer region is highly absorbed and quite softer than the inner region, with $\mathrm{N}_{H} \sim 16.2( \pm 5.2) \times 10^{22} \mathrm{~cm}^{-2}$ and $\mathrm{kT}=4.4( \pm 2.8) \mathrm{keV}$. Such characteristics are already observed in other X-ray plerions. Based on the morphological and spectral X-ray information, we confirm the SNR nature of G337.2+0.1, and suggest that the central region of the source is a pulsar wind nebula $(\mathrm{PWN})$, originated by an energetic though yet undetected pulsar, that is currently losing energy at a rate of $\sim 10^{36} \mathrm{erg} \mathrm{s}^{-1}$.
\end{abstract}

Subject headings: X-rays: individual (AX J1635.9-4719) - (ISM:) supernova remnants - ISM: individual (G337.2+0.1) — X-rays: ISM — radiation mechanisms: non-thermal 


\section{Introduction}

Supernova remnants (SNRs) of the Crab-like or plerionic class are objects characterized in the radio band by a compact, filled-center morphology with a relatively flat spectral index (Slane et al. 2000). In the X-ray band they present non-thermal spectra, characteristic of synchrotron emission. The non-thermal spectrum, in some cases, can even reach the very high-energy gamma-ray region, like in MSH 15-52, G18.0-07, and Vela X (see Aharonian et al. 2005, 2006a). It is widely believed that plerions are powered by the loss of rotational energy from energetic pulsars, although clear evidence of the presence of these objects is often lacking. The pulsar wind forms a nebula inside the SNR (the PWN), where relativistic particles can be efficiently accelerated producing synchrotron radiation that yields the typical morphologies observed at radio and X-rays.

The new generation of X-ray instruments with improved sensitivity and spatial/spectral resolution like ASCA, XMM, CHANDRA and INTEGRAL allows to detect and study not only distant energetic objects with these characteristics, but also those located in regions of high density.

Recently, Combi et al. (2005) have presented evidence supporting a SNR origin for the radio source G337.2+0.1. The object was discovered by the MOST galactic plane survey at 843-MHz towards the Norma spiral arm (Whiteoak \& Green 1996), and later, detected with the ASCA telescope during a survey of part of the galactic plane (Sugizaki et al. 2001). Its integrated flux was reported to be $\sim 1.2 \times 10^{-12} \mathrm{erg} \mathrm{cm}^{-2} \mathrm{~s}^{-1}$ in the $0.7-10 \mathrm{keV}$ energy band. The photon index is poorly constrained: $\Gamma=2.8_{-1.6}^{+2.6}$, and the absorption column density of the best-fit model yielded $\mathrm{N}_{H}=15_{-9}^{+15} \times 10^{22} \mathrm{~cm}^{-2}$ (Combi et al. 2005). A thorough study of its radio (continuum and line) and $\mathrm{X}$-ray properties shows that the emission from the source is consistent with what is expected for a young SNR located at a distance $d \sim 14 \mathrm{kpc}$ (Combi et al. 2005). More recently, G337.2+0.1 has been suggested as the potential counterpart of the high-energy gamma-ray source HESS J1634-472 (Aharonian et al. 2006b): this possibility needs further confirmation. Throughout this paper, we adopt $14 \mathrm{kpc}$ as the distance to G337.2+0.1 (hence, 1' corresponds to 4 pc) .

In this Letter, we present the first XMM-Newton observations of the SNR candidate G337.2+0.1. Based on its X-ray properties we are able to confirm that this object is a nonthermal SNR with hard, featureless power-law spectrum and, possibly, a PWN originated by a non-detected energetic pulsar. In Section 2, we present the X-ray observations and data reduction. X-ray analysis and results are presented in Section 3, and in Section 4 we provide a brief discussion and a summary. 


\section{X-ray Observations and data reduction}

The SNR candidate G337.2+0.1 has been marginally observed on February 2004 by the XMM-NewtonX-ray satellite in two separated observations (Obs-Id. 0204500201 and 0204500301). Both observations were centered towards the source IGR J16358-4726 $\left(\alpha_{\mathrm{J} 2000.0}=\right.$ $16^{\mathrm{h}} 35^{\mathrm{m}} 53.820, \delta_{\mathrm{J} 2000.0}=-47^{\circ} 25^{\prime} 41^{\prime \prime} .10$ ), and were acquired with the EPIC MOS (Turner et al. 2001) and EPIC PN (Strüder et al. 2001) cameras. Observations were taken with a "thin" filter, and in the full frame (FF) imaging mode. Temporal resolution is $2.5 \mathrm{~s}$ and $200 \mathrm{~ms}$ for the MOS and PN CCDs, respectively ${ }^{1}$. Observations were obtained from the XMM-Newton Science Archive $(\mathrm{XSA})^{2}$, and raw EPIC data were calibrated using the last version of the Standard Analysis System (SAS) ${ }^{3}$. To create images, spectra and light curves, we selected events with FLAG 0, and PATTERNS $\leq 12$ and 4 for MOS and PN cameras, respectively.

The off-angle of G337.2+0.1 respect to the center of the observation is about $\sim 6.67$ arcmin, which implies a reduction of the XMM-Newton effective area of about $12 \%$. The net exposure times of the observations are 34.7 (Obs-Id. 0204500201) and 32.6 (Obs-Id. 0204500301) ks. Unfortunately, the first observation was affected by a high and variable soft proton background level (Lumb et al. 2002), whereas the second one (Id. 0204500301) is unaffected by background fluctuations. We derive Good Time Intervals (GTI) by the accumulation of background light-curves in the 10-15 keV energy band, which leads to a reduction of $\sim 87 \%$ in the net exposure time of the Obs-Id. 0204500201. In order to avoid contamination for high background patterns hereafter our analysis concerns only to the observation 0204500301. The number of detected counts in the 0.5-2.5 and 2.5-10.0 keV energy bands are 117/121/315 and 431/403/1154 for the MOS1, MOS2 and PN cameras, respectively. Finally, at the SNR G337.2+0.1 EPIC-PN position there is a CCD gap in the $\mathrm{X}$-ray image, leading us to ignore these data only for the X-ray image analysis section, but they are included for the rest of our study.

\footnotetext{
${ }^{1}$ http://xmm.vilspa.esa.es/external/xmm_user_support/documentation/

${ }^{2} \mathrm{http}: /$ xmm.vilspa.esa.es/xsa/

${ }^{3} \mathrm{http}: / / \mathrm{xmm}$.vilspa.esa.es/external/xmm_sw_cal/sas.shtml
} 


\section{X-ray analysis of G337.2+0.1}

\subsection{Image}

The coordinates of the SNR G337.2+0.1 were defined at the position where X-ray emission peaks $\left(\alpha_{\mathrm{J} 2000.0}=16^{\mathrm{h}} 35^{\mathrm{m}} 54^{\mathrm{s}} .95, \delta_{\mathrm{J} 2000.0}=-47^{\circ} 19^{\prime} 02^{\prime \prime} .2\right)$, being the errors in R.A. and DEC of $\epsilon_{\alpha}= \pm 2$ s. 1 and $\epsilon_{\delta}= \pm 3^{\prime \prime} .3$, respectively (at the $90 \%$ of confidence). This position agrees well with the previous estimate of the radio position (Combi et al. 2005), but differs in $\sim 50$ arcsec from the ASCA coordinates. Because of the poor spatial resolution $(\sim 2.9$ arcmin) the ASCA telescope alone is not conclusive in resolving the SNR G337.2+0.1. Fortunately, the availability of the $0.5-10.0 \mathrm{keV}$ X-ray observations from XMM-Newton, the largest X-ray telescope so far $\left(1480 \mathrm{~cm}^{2} @ 1.5 \mathrm{keV}\right)$, allows to investigate with success the $\mathrm{X}$-ray nature of this source. In fact, $X M M-N e w t o n$ has $\sim 40$ times more spatial resolution than ASCA, whereas the sensitivity limit is at least $\sim 3$ times better.

Figure 1 shows the X-ray image of the SNR G337.2+0.1 in the $0.5-10.0 \mathrm{keV}$ energy band. The image does not reveal a typical rim-brightened outer SNR shell, so the overall size of the diffuse X-ray emission is uncertain.

We use the clean event files to generate MOS1 ans MOS2 images in the energy band [0.5-10] keV with a spatial binning of 4.35 arcsec per pixel. In order to increase the signalto-noise $(\mathrm{S} / \mathrm{N})$ ratio, we use the emosaic SAS task to merge together the two images. The corresponding set of exposure maps for each camera has been prepared to account for spatial quantum efficiency and mirror vignetting by running the SAS task eexmap. Exposure vignetting corrections were performed by dividing the superposed count image by the corresponding superposed exposure maps. We adaptively smoothed this image to a $\mathrm{S} / \mathrm{N}$ ratio of 10 using the SAS task asmooth (see Figure 1). Plotted contours correspond to 1, 2, 3 and 4 sigma levels over the mean background flux of the image $\left(\sim 6 \times 10^{-5} \mathrm{ph} / \mathrm{cm}^{2} / \mathrm{s}\right)$.

Finally, we are able to investigate the spatial extent of G337.2+0.1. Initially, this was performed by Combi et al. (2005) using ASCA data. However, the low resolution of this image led these authors to consider a radial analysis at large angular distance $(\sim 10 \operatorname{arcmin})$. Thanks to the high resolution XMM-Newton image, we found at least 19 weak point-like X-ray sources inside this radius, making the ASCA studies of G337.2+0.1 to be hardly biased by source contamination effects. According to the image presented in Figure 1, G337.2+0.1 does not extend farther than 1.5 arcmin from the central peak. We also compare G337.2+0.1 spatial extent with that produced by a point source placed at a similar off-axis $(\sim 6 \operatorname{arcmin})$ position. In Figure 2 we show that the SNR G337.2+0.1 has an extension $~ 3.5$ times larger than what is expected for a point-like source. 


\subsection{Spectral analysis}

For the spectral analysis we used MOS and PN data. It was performed using the XSPEC package (Arnaud 1996). Since the statistics of the source is not complete enough to perform a spatial-spectral analysis, we extracted X-ray photon events from only three different regions: i) a circular region of 50 arcsec; ii) a circular region of only 12 arcsec that accounts for the central source observed in the image; iii) an annulus for the extended emission of inner-outer radii of 12-50 arcsec. The background region was taken from a nearby blank region in the neighborhood of the source. Ancillary response files (ARFs) and redistribution matrix files (RMFs) were calculated. All spectra are grouped with a minimum of 16 counts per bin. The background-subtracted spectra of the MOS and PN data (the upper line) are shown in Figure 3. We checked for possible background contamination in our spectra, inconsistency in the extraction of local backgrounds and differences in background corrected spectra of SNR G337.2+0.1. We note excesses in spectra, essentially at energies between 7.5 to $8 \mathrm{keV}$. However, such features have a low statistical significance $(\sim 1$ to 1.5 sigma $)$ and are related to fluorescence lines in the background spectrum of the XMM-Newton (e.g. De Luca \& Molendi 2004).

Our analysis of the XMM-Newton EPIC spectra was essentially performed using a single non-thermal model, described by a simple Power-Law emission model. We also fit the spectra by a thermal emission model (APEC, Brickhouse 2003). Both models were affected by an absorption ISM component (WABS, Morrison \& McCammon 1983). The goodness of the model fit was derived according to the $\chi^{2}$-test statistics. The best fitting parameters of the models are shown in Table 1 , and the errors quoted are $90 \%$ confidence limits.

According to the results presented in Table 1, the central part of the SNR appears quite harder $(\Gamma \sim 0.96)$ than the outer one $(\Gamma \sim 2.38)$. We suggest that the most reasonable interpretation of observed emission from the central part of SNR is synchrotron radiation from relativistic electrons accelerated in the vicinity of the central source of the SNR.

The softening of the spectrum toward the outer regions of the nebula is a well known effect which has been seen in other X-ray plerions (e.g., G0.9+0.1, Porquet et al. 2003; 3C58, Torii et al. 2000; G21.5-0.9, Slane et al. 2000).

To get a statistical assessment of the X-ray variability of the SNR G337.2+0.1, we use the 32.6-ksec EPIC-PN observation to compare the time arrival distribution of source photons by means of the Kolmogorov-Smirnov (KS) test (Press et al. 1992). We use an extraction region centered in the SNR with a radii of 50 arcsec. The total number of photons is 1470. We see no significant pulsed signal with a period greater than twice the read-out time of the EPIC-PN camera in the FF mode, which corresponds to a Nyquist limit of $400 \mathrm{~ms}$. 


\section{Discussion}

The X-ray morphology of G337.2+0.1 shows a centrally peaked emission, surrounded by a diffuse X-ray nebula. The lightcurve of the object does not show any significant flux variability above $0.4 \mathrm{seg}$, implying that at first glance, a pulsar origin for the central contribution could be ruled out. However, a detailed spectral analysis indicates that the outer region is softer than the inner region, a phenomenon observed previously in several X-ray plerions with PWN (e.g., IC443, Bocchino \& Bykov 2001). A spectral analysis of the central component of the SNR shows that the X-ray spectrum is well represented by a single power-law with a photon index $\Gamma=0.96 \pm 0.56$, a value similar to that of objects powered by an energetic pulsar (Gotthelf 2003). Moreover, the non-detection of line emission in this spectrum is consistent with synchrotron radiation from a population of relativistic electrons. These facts suggest a non-thermal origin for the X-ray emission. We therefore conclude that the system G337.2+0.1/ AX J1635.9-4719 is a non-thermal SNR with, possibly, a non-detected pulsar.

Possible reasons for the non-detection of a pulsar inside the SNR are a short rotation period (less than $400 \mathrm{~ms}$ ) or unfavorable geometrical conditions. The presence of a pulsar is suggested by the central X-ray peak found inside G337.2+0.1. In what follows we explore the possibility that there exists a hidden pulsar-powered component (plerion) within the SNR. Using the empirical formula derived by Seward \& Wang (1988), $\log L_{\mathrm{X}}\left(\mathrm{erg} \mathrm{s}^{-1}\right)=1.39 \log \dot{E}-$ 16.6, where $L_{\mathrm{X}}$ is the X-ray luminosity of the plerion in the $0.2-4 \mathrm{keV}$ band, we can make an estimate of the spin-down luminosity of the pulsar (see, also, Becker \& Trümper 1997). Using the X-ray flux of the compact source and its nebula, $F_{\mathrm{X}}(0.2-4 \mathrm{keV})=4.9 \pm 1.7 \times 10^{-13} \mathrm{erg} \mathrm{s}^{-1}$ $\mathrm{cm}^{-2}$, we get $L_{\mathrm{X}}=1.1 \times 10^{34} \mathrm{erg} \mathrm{s}^{-1}$ (unabsorbed). This implies a spin-down luminosity of $\dot{E} \sim 2.5 \times 10^{36} \mathrm{erg} \mathrm{s}^{-1}$, and a period of $P \geq 0.08\left(t_{3} \dot{E_{38}}\right)^{1 / 2}(\mathrm{~s})$, where $\dot{E}_{38}$ is the spin-down luminosity in units of $10^{38} \mathrm{erg} \mathrm{s}^{-1}$, and $t_{3}$ is the pulsar age in units of $10^{3}$ years. In order to compare this result with those obtained with other empirical relations between the X-ray luminosity and the rate of the spin-down energy loss, we have used the Becker \& Trümper (1997) and Possenti et al. (2002) equations. In the first case, (taking into account only the Xray flux of the point source in the $0.1-2.4 \mathrm{keV}$ band) the spin-down luminosity is $\dot{E} \sim 3 \times 10^{35}$ erg s $\mathrm{s}^{-1}$, a factor 9 lower than the value obtained with the Seward \& Wang (1988) relation. In the second case (using the X-ray flux of the compact source and its nebula in the $2-10 \mathrm{keV}$ band), we got $\dot{E} \sim 8 \times 10^{36} \mathrm{erg} \mathrm{s}^{-1}$, a value that is a factor 3 higher than the value obtained with the Seward \& Wang (1988) relation. On average, a value $\sim 10^{36} \mathrm{erg} \mathrm{s}^{-1}$ seems to be reasonable. If we assume a pulsar period of less than $0.4 \mathrm{~s}$, we obtain an upper limit for the age of the pulsar of $t \leq 1000$ years.

We have seen that G337.2+0.1 does not show a rim-brightened outer SNR shell. This could be the result of the absorption of the soft thermal emission from the forward shock 
by the very high absorbing column density. Other sources like Crab, G21.5-0.9 (Slane et al. 2000) and 3C58 (Torii et al. 2000) have weak or absent X-ray rims and all are powered by young X-ray pulsars (Murray et al. 2002; Camilo et al. 2006).

It could be interesting to compare the characteristics of G337.2+0.1 with 3C58. The X-ray luminosities, between 0.5 and $10.0 \mathrm{keV}$, are $\sim 4.8 \times 10^{34} \mathrm{erg} \mathrm{s}^{-1}$ and $\sim 2.4 \times 10^{34} \mathrm{erg}$ $\mathrm{s}^{-1}$, respectively. The radio luminosities, at $1 \mathrm{GHz}$, are $\sim 3 \times 10^{32} \mathrm{erg} \mathrm{s}^{-1}$ and $\sim 4 \times 10^{32}$ $\operatorname{erg~s}^{-1}$. We see, then, that both sources are quite similar. We notice that the estimated age of 3C58 is $\sim 800 \mathrm{yr}$, a value based on the association of the SNR with the supernova 1181 (Stephenson \& Green 2002). The nature of this SNR is discussed by Camilo et al. (2006), who, however, argue against the association with this historical SN (Bietenholz et al. 2001). The most significant difference seems to be the absence of any thermal component in the case of G337.2+0.1. If we compare with the Crab, on the contrary, we see that the Crab pulsar is injecting around two orders of magnitude more energy per time unit in the nebula than G337.2+0.1. The spin-down luminosity inferred for the pulsar from the new X-ray data sets an upper limit to the energy available for high-energy cooling channels like inverse Compton scattering and proton-proton interactions. The luminosity of the nearby HESS source J1634-472 ( $E>1 \mathrm{TeV})$, if it is located at the same distance inferred for G337.2+0.1, would be $L_{\gamma} \sim 7 \times 10^{34} \mathrm{erg} \mathrm{s}^{-1}$. So, a physical association would be possible only if $\sim 7 \%$ of the spin-down luminosity is converted in high-energy $\gamma$-rays.

Complementary studies of the PWN scenario will involve high-resolution X-ray observations with Chandra satellite, and radio observations with ATCA, to allow the comparison of the X-ray spectrum and morphology with those at the radio band. GLAST observations could reveal a GeV $\gamma$-ray source if the proposed association with HESS J1634-472 is correct.

We thank an anonymous referee for detailed and very constructive comments on the manuscript. J.A.C. is a researcher of the programme Ramón y Cajal funded jointly by the Spanish Ministerio de Ciencia y Tecnología and Universidad de Jaén. J.A.C. acknowledges support by DGI of the Spanish Ministerio de Educación y Ciencia under grant AYA200407171-C02-02, FEDER funds and Plan Andaluz de Investigación of Junta de Andalucía as research group FQM322. J.F.A.C acknowledges support by the Marie Curie Fellowship Contract N MTKD-CT-2004-002769 on the project "The influence of Stellar High Energy Radiation on Planetary Atmospheres". G.E.R. and P.B. were supported in part by grant BID 1728/OC-AR PICT 03-13291 (ANPCyT) and CONICET (PIP 5375). 


\section{REFERENCES}

Aharonian, F., et al. 2005, A\&A, 442, L25.

Aharonian, F., et al. 2006a, A\&A, 448, L43.

Aharonian, F., et al. 2006b, ApJ, 636, 777.

Anders, E., \& Grevesse, N. 1989, Geochim. Cosmochim. Acta, 53, 197.

Arnaud, K. A. 1996, ASP Conf. Ser. 101: Astronomical Data Analysis Software and Systems $\mathrm{V}, 101,17$.

Becker, W., \& Truemper, J. 1997, A\&A, 326, 682

Bietenholz, M. F., Kassim, N. E., \& Weiler, K. W. 2001, ApJ, 560, 772

Bocchino, F., \& Bykov, A. M. 2001, A\&A, 376, 248.

Brickhouse, N. S. 2003, IAUJD, 17, 23.

Camilo, F., et al. 2006, ApJ, 637, 456

Combi, J.A., Benaglia, P., Romero, G.E., Sugizaki, M., 2005, A\&A, 431, L9.

De Luca, A. \& Molendi, S. 2004, 419, 837

Gotthelf, E. V. 2003, ApJ, 591, 361

Lumb, D. H., Warwick, R. S., Page, M., \& De Luca, A. 2002, A\&A, 389, 93.

Morrison, R., \& McCammon, D. 1983, ApJ, 270, 119.

Murray, S. S., et al. 2002, ApJ, 568, 226

Porquet, D., Decourchelle, A., \& Warwick, R. S. 2003, A\&A, 401, 197 .

Possenti, A., Cerutti, R., Colpi, M., Mereghetti, S. 2002, A\&A, 387, 993

Press, W. H., Teukolsky, S. A., Vetterling, W. T., \& Flannery, B. P. 1992, Cambridge: University Press, - c1992, 2nd ed.

Seward, F. D., \& Wang, Z.-R. 1988, ApJ, 332, 199.

Slane, P., Chen, Y., Schulz, N. S., et al. 2000, ApJ, 533, L29. 
Stephenson, F. R., \& Green, D. A. 2002, Historical Supernovae and their Remnants (Oxford: OUP)

Strüder, L., et al. 2001, A\&A, 365, L18.

Sugizaki, M., et al. 2001, ApJS, 134, 77.

Torii, K., Slane, P. O., Kinugasa, K., Hashimotodani, K., \& Tsunemi, H. 2000, PASJ, 52, 875.

Turner, M. J. L., et al. 2001, A\&A, 365, L27.

Whiteoak, J. B. Z. \& Green, A. J. 1996, A\&A, 118, 329.

Yamaguchi, H., Ueno, M., Koyama, K., et al. 2004, PASJ, 56, 1059 
Table 1: X-ray spectral parameters of the SNR G337.2+0.1

\begin{tabular}{l|ll|ll|ll}
\hline \multirow{2}{*}{$\begin{array}{l}\text { Region: } \\
\text { Model: }\end{array}$} & \multicolumn{2}{|c|}{ All } & \multicolumn{2}{c|}{ Inner } & \multicolumn{2}{c}{ Outer } \\
\cline { 2 - 7 } & Power-law & APEC & Power-law & APEC $(\dagger)$ & Power-Law & APEC \\
\hline $\mathrm{N}_{H}\left(\mathrm{~cm}^{-2}\right)$ in $\left[10^{22}\right]$ & $11.49 \pm 2.73$ & $11.17 \pm 2.18$ & $5.9 \pm 1.52$ & $6.45 \pm 2.31$ & $16.21 \pm 5.62$ & $16.26 \pm 5.25$ \\
$\Gamma \mid \mathrm{kT}(\mathrm{keV})$ & $1.82 \pm 0.45$ & $10.01 \pm 6.49$ & $0.96 \pm 0.56$ & $(64 \pm 64)$ & $2.38 \pm 0.78$ & $4.38 \pm 2.79$ \\
Abundance & --- & $<0.05$ & --- & $<0.05$ & --- & $<0.05$ \\
Norm. in $\left[\times 10^{-4}\right]$ & $4.91 \pm 1.93$ & $8.28 \pm 2.83$ & $0.55 \pm 0.51$ & $(0.83 \pm 2.4)$ & $4.01 \pm 1.44$ & $6.7 \pm 2.81$ \\
Flux $\left(\mathrm{erg} \mathrm{s}^{-1} \mathrm{~cm}^{-2}\right)$ & $11.7_{8.8}^{12.9}$ & $9.81_{6.2}^{11.1}$ & $1.3_{1.2}^{2.1}$ & $1.1_{0.9}^{1.4}$ & $8.2_{7.2}^{9.9}$ & $7.4_{6.8}^{9.3}$ \\
Reduced $\chi_{\nu}^{2}$ & 1.13 & 1.14 & 1.10 & 1.25 & 1.09 & 1.06 \\
\hline
\end{tabular}

Notes: Flux is absorption-corrected in units of $10^{-13}\left(\mathrm{erg} \mathrm{s}^{-1} \mathrm{~cm}^{-2}\right)$ calculated in the (0.5-10.0 $\mathrm{keV})$ energy range. Normalization was calculated according to: $10^{-14} / 4 \pi r^{2}(1-z)^{2} \int n_{e} n_{H} d V$. $\dagger$ APEC thermal model could not fit the spectra in a consistent way, yielding very ill-constrained parameters as solution. The non-thermal (power-law) fit seems to be the most representative emission model for the central part of the SNR. $\ddagger$ The abundance was adopted from Anders \& Grevesse (1989) and left as free parameter along all our fits, but its value is hardly affected by the low statistic of the spectra. 


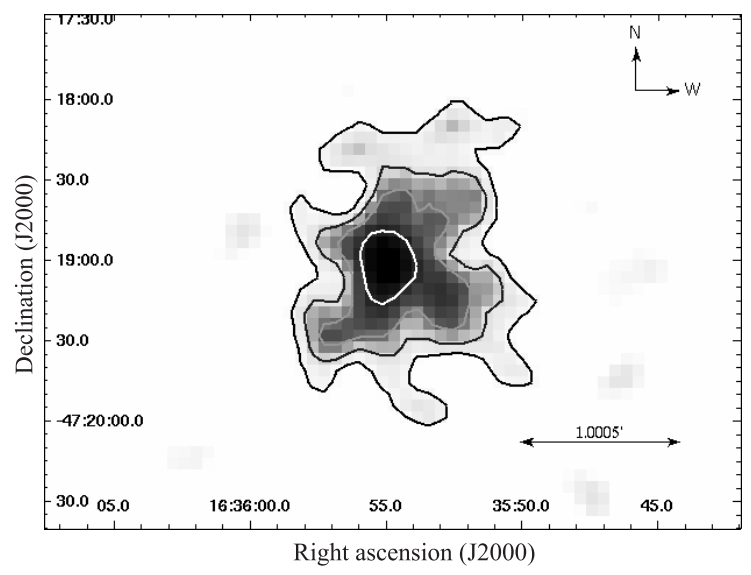

Fig. 1. - X-ray image of the G337.2+0.1 in the $0.5-10.0 \mathrm{keV}$ band. Typical local background level of the smoothed image is $\sim 0.94( \pm 0.17)$ counts $\mathrm{px}^{-1}$. Contours show the level of $1.6(\sim 1 \sigma), 3.2(\sim 2 \sigma), 4.7(\sim 3 \sigma)$ and $6.3(\sim 4 \sigma)$ photons $\mathrm{px}^{-1}$, from outer to inner curves, respectively. 


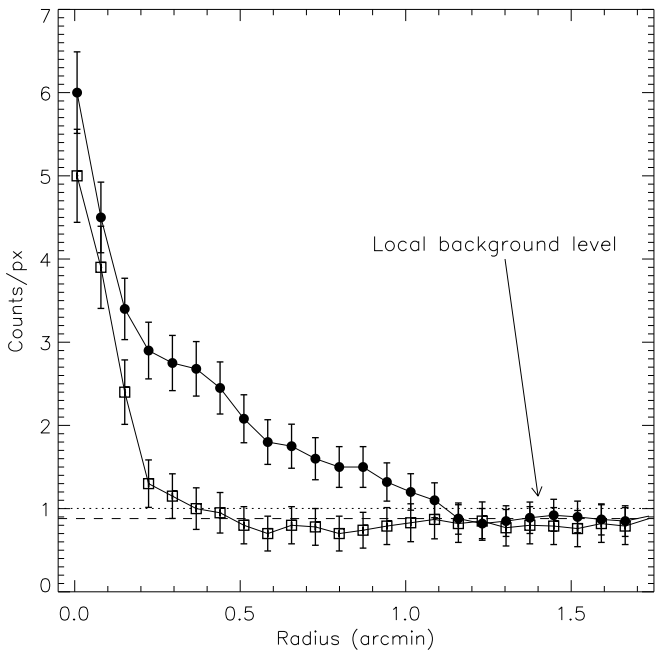

Fig. 2.- Radial profile of the smoothed X-ray image of SNR G337.2+0.1 (filled circles). Open squares refer to the radial profile of an observed point source located at roughly the same off-axis position. 

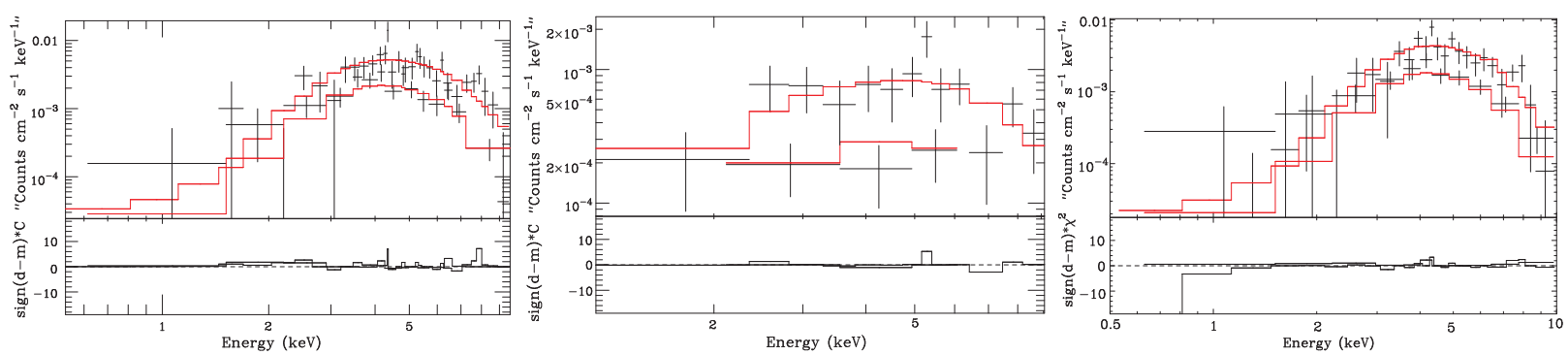

Fig. 3.- X-ray spectra of the SNR G337.2+0.1 in the 0.5-10.0 keV band. In all figures PN data are the upper line. Left: Spectrum extracted on the whole SNR. Center: Spectrum of the compact source observed in the center of SNR G337.2+0.1. Right: Outer spectrum that excludes the central contribution. 